\section{Commentary: Modified branch-first technique in thoracoabdominal aortic aneurysm repair: Does simpler mean safer?}

\author{
Thierry Carrel, MD, ${ }^{\mathrm{a}}$ and Martin Czerny, $\mathrm{MD}^{\mathrm{b}, \mathrm{c}}$
}

The genuine intention of thoracoabdominal aortic aneurysm (TAAA) repair is the restoration of a regular anatomy with a special attention to limit a potential end-organ ischemia to a minimum during repair. ${ }^{1}$ This journey began with a simple clamp-and-sew technique and developed later into very sophisticated approaches that include repair under left heart bypass or full cardiopulmonary bypass with mild, moderate, or deep hypothermia; distal exsanguination; and additional intermediate and combined strategies applying selective organ perfusion not only to visceral, renal, and the lower extremities arteries but also to the spinal cord. ${ }^{2,3}$ Not to mention that extracorporeal circulation represented the basis for the majority of these operative techniques.

On the other side, reducing perfusion time to a minimum is a welcome aspect because adverse outcomes have quite often been put in relationship to the duration of extracorporeal circulation. In the end, it remains clear that besides a perfect technical result, collateral injury of the procedure must be kept to a minimum and reducing the duration of perfusion is among the essential objectives in TAAA

From the ${ }^{a}$ Department of Cardiac Surgery, University Hospital Zürich, Zürich, Switzerland; ' ${ }^{\mathrm{b}}$ Department of Cardiovascular Surgery, University Heart Center, Freiburg-Bad Krozingen, Germany; and ${ }^{c}$ Faculty of Medicine, Albert Ludwigs University, Freiburg, Germany.

Disclosures: Dr Czerny is a consultant to Terumo Aortic, Medtronic, Endospan, and NEOS; has received speaking honoraria from Cryolife-Jotec and Bentley; and is shareholder of TEVAR Ltd. Dr Carrel reported no conflicts of interest.

The Journal policy requires editors and reviewers to disclose conflicts of interest and to decline handling or reviewing manuscripts for which they may have a conflict of interest. The editors and reviewers of this article have no conflicts of interest.

Received for publication March 31, 2021; revisions received March 31, 2021; accepted for publication April 6, 2021; available ahead of print April 15, 2021.

Address for reprints: Thierry Carrel, MD, Department of Cardiac Surgery, University Hospital Zurich, Rämistrasse 100, CH-8091, Zürich, Switzerland (E-mail: thierry. carrel@usz.ch).

JTCVS Techniques 2021;7:32-3

2666-2507

Copyright (C) 2021 The Author(s). Published by Elsevier Inc. on behalf of The American Association for Thoracic Surgery. This is an open access article under the CC BY-NC-ND license (http://creativecommons.org/licenses/by-nc-nd/4.0/).

https://doi.org/10.1016/j.xjtc.2021.04.001

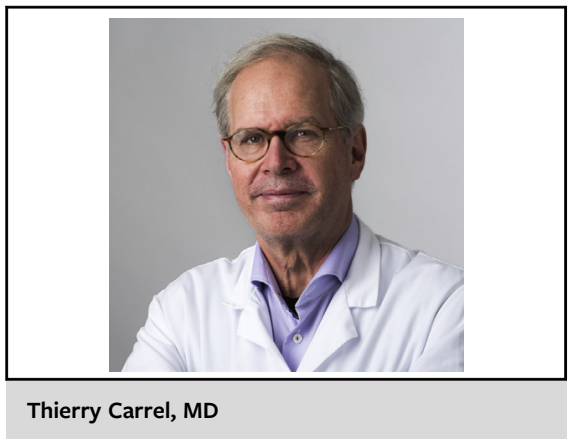

CENTRAL MESSAGE

Branch-first technique in TAAA repair may reduce duration of perfusion and visceral ischemia, but these advantages must be weighed against perfect length of the grafts and optimized hemostasis.

repair. ${ }^{4}$ The concept of visceral and renal reimplantation as the first step (rather than the last step, as the majority of surgeons probably undertake), is appealing. Nevertheless, the intention is always to keep ischemic time to a minimum, which can also be obtained by selective organ perfusion.

In their case report, the authors nicely demonstrate that their approach shortened end-organ ischemia as much as possible - which also contributed to achievement of among the main objectives of TAAA repair, namely quick restoration of regular anatomy. This approach should be kept in mind as a useful adjunct in the surgical armamentarium of organ protection in a highly complex field. However, some points deserve a special comment:

- Performing the anastomosis of the side branches with the visceral arteries outside of the aorta requires careful estimation of the definitive length of these side branches to avoid kinking.

- The visceral and/or renal branches may be more fragile when anastomosed this way rather than from inside of the aorta.

- The definitive length of the main aortic graft has to be determined with particular attention to avoid a too long or too short graft because all branches have been preanastomosed.

- This technique precludes the possibility to perform a tight inclusion of the prosthetic graft with a 
xeno-pericardial patch to optimize hemostasis at the end of the operation.

\section{References}

1. Hiremath N, Younes H, Aleinati T, Park WM. Open repair of extent-III thoracoabdominal aortic aneurysm using modified branch-first technique. J Thorac Cardiovasc Surg Tech. 2021;7:29-31.

2. Czerny M, Mach M, Schönhoff F, Basciani R, Jenni HJ, Carrel TP, et al. Double arterial perfusion strategy for extensive thoracic aortic surgery to avoid lower body hypothermic circulatory arrest. Eur J Cardiothorac Surg. 2014; 45:460-5.

3. Etz C, Weigang E, Hartert M, Lonn L, Mestres C, Di Bartolomeo R, et al. Contemporary spinal cord protection during thoracic and thoracoabdominal aortic surgery and endovascular aortic repair: a position paper of the vascular domain of the European Association for Cardio-Thoracic Surgery. Eur J Cardiothorac Surg. 2015; 47:943-57.

4. Jassar A, Kreibich M, Morlock J, Kondov S, Scheumann J, Kari FA, et al. Thoracoabdominal aortic replacement after thoracic endovascular aortic repair with large stent-grafts: diameter correction with modified use of the Vascutek ${ }^{\circledR}$ Siena ${ }^{\mathrm{TM}}$ collared branched graft. Ann Thorac Surg. 2018;105:587-91. 